\title{
The Development and Preliminary Validation of Chinese Nurses' Workplace Stressor Scale-30 (CNWSS-30)
}

\author{
Yeyun Yu1, Weiwei Zhu1, Ruiwei Shen², Lu Lv² \\ ${ }^{1}$ Wego Management Institution, Weihai, China \\ ${ }^{2}$ Hangzhou Yicheng Business Management \& Consulting Co., Ltd., Hangzhou, China \\ Email: yuyeyun@126.com
}

How to cite this paper: Yu, Y. Y., Zhu, W. W., Shen, R. W., \& Lv, L. (2020). The Development and Preliminary Validation of Chinese Nurses' Workplace Stressor Scale-30 (CNWSS-30). Psychology, 11, 1511-1518. https://doi.org/10.4236/psych.2020.1110096

Received: September 8, 2020

Accepted: October 19, 2020

Published: October 22, 2020

Copyright $\odot 2020$ by author(s) and Scientific Research Publishing Inc. This work is licensed under the Creative Commons Attribution International License (CC BY 4.0).

http://creativecommons.org/licenses/by/4.0/ (c) (i) Open Access

\begin{abstract}
Objective: The purpose of this study was to develop a concise and widely applicable Chinese nurse workplace stressor scale (CNWSS). Methods: 3895 Nurses were investigated by a questionnaire including 159 candidate items of nurse work stressors. The Automatic Thoughts Questionnaire (ATQ-30) and Scale of Medical Staff Resilience (SMSR) were used as validity criterion. 162 samples were further selected for the test-retest after 4 weeks. Results: By means of exploratory factor analysis (EFA), 30 items were finally extracted for CNWSS (CNWSS-30), including 6 subscales, namely, organizational role adaptability, workload, nurse-patient relationship, income and respect, self-efficacy, and responsibility. Moreover, confirmatory factor analysis (CFA) showed good fitness $(\mathrm{GIF}=0.90, \mathrm{AGFI}=0.89, \mathrm{CFI}=0.91$, RMSEA $=0.06)$. The results also found strong positive correlations among six subscales of CNWSS-30 with ATQ-30 ( $\mathrm{r}=0.15-0.34, p<0.01)$, and negative correlations with SMSR $(\mathrm{r}=-0.26--0.06, p<0.05)$. The internal consistency reliability coefficients showed high reliability (the total scale was 0.93 , subscales ranged from 0.66 to $0.91, p<0.01$ ), and the result of test-retest reliability (the total scale was 0.77 , subscales ranged from 0.60 to $0.76, p<0.01$ ) also revealed high stability. Conclusion: Generally, CNWSS-30 is reliable and valid, and can be used as a tool for measuring the Chinese nurse stressors at workplace.
\end{abstract}

\section{Keywords}

Chinese Nurse Stressors, Workplace, Scale, Localization

\section{Introduction}

Nursing is a significant part of modern medical industry, and the quality of 
nursing care is one of the important factors that determine whether a patient can recover smoothly. Evidences suggested nurse work stress might adversely impact the quality of nursing (e.g. Cimiotti, Aiken, Sloane, \& Wu, 2012; Arnetz, Sudan, Goetz, Counts, \& Arnetz, 2019), even to nurse mental health (e.g. Sun \& Wen, 2013; Lee \& Akhtar, 2011; Applebaum, Fowler, Fiedler, \& Osinubi, 2010; Khamisa, Peltzer, \& Oldenburg, 2013). To reduce the nurses' stress, hospital managements have used different methods to improve work environment (i.e. strengthen basic infrastructure construction), and professional skills and increase employee benefits (Cheng, Liu, Wu, \& Lu, 2015). However, organizational interventions are targeted at most but not all of these sources, and their effectiveness is likely to be limited, at least in the short to medium term (Mcvicar, 2003). Therefore, for reducing nurses' stress effectively and accurately, it is very important to identify and recognize the nurse work stressors, which is the first step in stress management process.

Work stress, defined as "the harmful physical and emotional responses that occur when the requirements of the job do not match the capabilities, resources, or needs of the worker" (NIOSH, 1999), has been perceived with negative implications for nurses' health, including burnout, compassion fatigue, depression, and anxiety. It is also associated with negative patient outcomes, like dissatisfaction, fails, and medication errors (Arnetz et al., 2019). Numbers of studies focused on these stress-related problems and investigated the stressful events for nurses. National Institute for Occupational Safety and Health pointed that nurse work stress was considered relevant with the following factors: work overload, time pressure, lack of social support at work, exposure to infectious diseases, needle injuries, exposure to work-related violence or threats, sleep deprivation, role ambiguity and conflicts, understaffing, career development issues, and dealing with difficult or seriously ill patients (NIOSH, 2008). Gray-Toft \& Anderson (1981) first conducted factor analysis of nurse stress and 7 factors were obtained, namely, death and dying, conflict with physicians, inadequate preparation in dealing with the emotional needs of patients and their families, lack of staff support, conflict with other nurses and supervisors, excessive workload, and uncertainty concerning the treatment of patient. Wheeler \& Riding (1994) analyzed the work stressors of nurses, and found 4 factors as results: workload against time pressure, organizational and management problems, poor interpersonal relationship, and poor working conditions/facilities. The existing nurse work stressors scale in China was translated and revised by the two scales above (e.g. Li, \& Liu, 2000; Yu, 2007; Yuan, Yu, \& Xu, 2007; Wang \& Meng, 2007). The scales introduced from abroad are all compiled based on the national psychological characteristics and condition, not fully applicable to other countries (Tong, 2002). Since there is a vast territory and diverse cultures in China, the translated scale may have a problem of cultural equivalence and, the items of existing scale were also relatively old. When verified reliability and validity, the sampling site was single; there was also no unified factor structure for each scale.

Due to the importance of these issues, this study selected Chinese nurses as 
participants, to develop the Chinese Nurses' Workplace Stressor Scale (CNWSS), which would be suitable for Chinese culture and the current nursing status in China.

\section{Method}

\subsection{Participants and Design}

From April to August of 2018, using the method of convenient sampling, 3895 nurses (3675 females and 220 males) were selected as participants. The random samples were taken from 4 different hospitals based on different administrative divisions in China. Participants aged from 20 to 62 years $(M=30.61, S D=6.51)$. 2929 nurses had bachelor degree, 940 nurses had below degree level and 62 nurses with the above degree level. For the professional titles there were 1687 nurses, 1572 practitioners, 434 nurses-in-charge, 180 associated chief nurse and 22 chief superintendent nurses. The exploratory factor analysis was performed on these 3895 nurses to explore the factor structure of CNWSS.

Furthermore, based on professional title, 1947 nurses extracted from 3895 nurses were assessed by confirmatory factor analysis (CFA). Finally, 162 samples were selected (151 females and 11 males, their age ranged from 22 to 58 years, $M$ $=31.90, S D=2.13)$ as participants to analyze the test-retest reliability after 4 weeks.

\subsection{Scale Development, Candidate Items Selection}

From April to May of 2016, 122 experienced head nurses from ten large 3a hospitals were selected as participants, and the open-ended questionnaires was investigated to collect the work stressor relevant items. 1178 related items were obtained. According by analyzing these items by four nurse experts and psychologists, then a selection of 159 items that represented best the 13 dimensions of work stressors were finally obtained. The items were using a 5-point Likert scale by self-reported, which represents the differences in the intensity, frequency and other aspects of items.

\subsection{The Automatic Thoughts Questionnaire (ATQ-30)}

ATQ-30 was developed by Hollon and Kendall (1980). It contains a 30-item instrument that measures the frequency of automatic negative statements about self. Participants ranked certain statements with a 5-point scoring $(1=$ none, $2=$ occasionally, $3=$ sometimes, $4=$ often, $5=$ persistent), which carries high reliability and validity. The translated version of the "Handbook of mental health scale for the updated version" was used in this study (Wang, Wang, \& Ma, 1999).

\subsection{Scale of Medical Staff Resilience (SMSR)}

SMSR is a local instrument for nurse resilience in Chinese culture context, designed by Dong in 2014, which consists of 6 subscales (24 items), including 
self-control, social support, family support, future prospect, tolerance, and competence. Participants ranked certain statements with a 5-point continuum from "extremely disagree" to "extremely agree", which carries high reliability and validity (Dong \& Qin, 2014). Therefore, we used SMSR to confirm the validity of CNWSS.

\section{Results}

By means of EFA, the number of items was reduced to 30 items according to the factor loadings (excluded factor loadings below 0.4). As a result, the CNWSS was consist of 6 subscales (totally 30 items): organizational role adaptability, workload, nurse-patient relationship, income and respect, self-efficacy, and responsibility, presented in Table 1.

To confirm the factor structure of CNWSS-30, CFA was performed and the results showed good fitness $(\mathrm{GIF}=0.897, \mathrm{AGFI}=0.887, \mathrm{CFI}=0.907, \mathrm{RMSEA}=$ 0.061). By correlation analysis, significant correlations were observed among CNWSS-30, ATQ-30, and SMSR factors. Specifically, there were strong positive correlations among six subscales (i.e. organizational role adaptability, workload, nurse-patient relationship, income and respect, self-efficacy, responsibility) of CNWSS-30 with ATQ-30 ( $\mathrm{r}=0.31,0.31,0.28,0.26,0.34,0.15, p<0.01)$, and negative correlations among the six subscales of CNWSS-30 with SMSR ( $\mathrm{r}=$ $-0.21,-0.26,-0.18,-0.18,-0.24,-0.06, p<0.05)$. The results are indicated in Table 2 .

Table 1. Results of EFA.

\begin{tabular}{|c|c|c|c|c|c|c|c|c|}
\hline \multirow{2}{*}{ Factors } & \multirow{2}{*}{$\begin{array}{c}\text { Item } \\
\text { Number }\end{array}$} & \multirow{2}{*}{ Items } & \multicolumn{6}{|c|}{ Factor Loading } \\
\hline & & & F1 & F2 & F3 & F4 & F5 & F6 \\
\hline \multirow{6}{*}{$\begin{array}{l}\text { Organizational } \\
\text { Role Adaptability }\end{array}$} & 104 & My leader isn't good at listening & 0.872 & 0.024 & -0.076 & 0.016 & -0.133 & -0.029 \\
\hline & 140 & My leader's demands are often biased & 0.765 & -0.012 & 0.065 & 0.093 & -0.089 & 0.002 \\
\hline & 89 & My leader doesn't understand and support me enough & 0.735 & -0.015 & 0.030 & 0.127 & -0.024 & -0.026 \\
\hline & 17 & It's difficult to get along with my leader & 0.659 & 0.101 & -0.066 & -0.008 & 0.004 & 0.008 \\
\hline & 94 & I often disagree with my leader & 0.625 & -0.108 & 0.016 & 0.020 & 0.068 & 0.059 \\
\hline & 63 & I often don't understand my leader's intentions & 0.524 & 0.047 & 0.026 & -0.040 & 0.225 & -0.012 \\
\hline \multirow{7}{*}{ Workload } & 56 & My life is irregular because of my work & -0.047 & 0.831 & 0.068 & -0.052 & -0.031 & -0.099 \\
\hline & 55 & Holidays cannot be normal rest & 0.028 & 0.676 & -0.005 & -0.040 & 0.004 & -0.004 \\
\hline & 157 & I couldn't spend my time freely & 0.126 & 0.652 & -0.074 & -0.054 & -0.028 & 0.173 \\
\hline & 77 & I have no time to sport & -0.053 & 0.630 & -0.124 & 0.089 & 0.021 & 0.097 \\
\hline & 66 & I don't have time and energy to take care of my family & -0.090 & 0.587 & -0.046 & 0.160 & 0.118 & 0.027 \\
\hline & 107 & I feel a little weak recently & 0.055 & 0.537 & -0.012 & 0.197 & 0.044 & -0.024 \\
\hline & 44 & I am suffering from sub-health & -0.034 & 0.496 & -0.076 & 0.277 & 0.009 & 0.190 \\
\hline \multirow{3}{*}{$\begin{array}{l}\text { Nurse-Patient } \\
\text { Relationship }\end{array}$} & 12 & Patients always make unreasonable requests & -0.056 & -0.183 & 0.888 & -0.017 & -0.071 & 0.214 \\
\hline & 58 & $\begin{array}{l}\text { I always couldn't get understanding and respect from } \\
\text { patients and their family }\end{array}$ & 0.002 & 0.141 & 0.642 & 0.152 & 0.023 & -0.249 \\
\hline & 113 & Patients and their family are always uncooperative & 0.064 & 0.058 & 0.603 & 0.071 & 0.069 & -0.053 \\
\hline
\end{tabular}






The accumulative variance contribution was $54.42 \%$.

Table 2. Correlations among the six subscales of CNWSS-30, ATQ-30 and SMSR $(n=1182)$.

\begin{tabular}{|c|c|c|c|c|c|c|c|}
\hline & Workload & $\begin{array}{l}\text { Nurse-Patient } \\
\text { Relationship }\end{array}$ & $\begin{array}{c}\text { Income and } \\
\text { Respect }\end{array}$ & Self-ability & Responsibility & ATQ-30 & SMSR \\
\hline Organizational Role Adaptability & $0.42^{\star *}$ & $0.40^{* *}$ & $0.42^{\star *}$ & $0.32^{* *}$ & $0.35^{\star *}$ & $0.31^{* *}$ & $-0.21^{* *}$ \\
\hline Workload & & $0.49^{* *}$ & $0.62^{\star *}$ & $0.19^{* *}$ & $0.57^{\star *}$ & $0.31^{* *}$ & $-0.26^{* *}$ \\
\hline Nurse-Patient Relationship & & & $0.62^{\star *}$ & $0.16^{* *}$ & $0.36^{* *}$ & $0.28^{\star *}$ & $-0.18^{* *}$ \\
\hline Income and Respect & & & & $0.13^{\star *}$ & $0.41^{* *}$ & $0.26^{* *}$ & $-0.18^{* *}$ \\
\hline Self-efficacy & & & & & $0.17^{\star *}$ & $0.34^{* *}$ & $-0.24^{* *}$ \\
\hline Responsibility & & & & & & $0.16^{* *}$ & $-0.06^{*}$ \\
\hline
\end{tabular}

${ }^{\star *} p<0.01,{ }^{*} p<0.05$.

The internal consistency reliability coefficients were also showed high reliability. Cronbach's alpha coefficient was 0.93 for the total scale (30 items). For the subscales of organizational role adaptability, workload, nurse-patient relationship, income and respect, self-efficacy, the Cronbach's alpha coefficients were $0.91,0.90,0.85,0.81,0.82,0.66$, respectively. The results of the test-retest reliability revealed strong reproducibility of CNWSS-30 and subscales (total scale was 0.77 , subscales result ranged from 0.60 to $0.76, p<0.01$ )

In addition, the participants were divided into two groups (high vs. low) according to the average of ATQ-30 scores. The results demonstrated that the high score group had higher score at all six subscales of CNWSS than the low score group ( $t$ value ranged from -10.18 to $-3.48, p<0.01$ ) (Table 3 ). 
Table 3. Comparison of CNWSS-30 in different depressive tendencies $(n=1182)$.

\begin{tabular}{ccccc}
\hline & \multicolumn{2}{c}{ ATQ-30 } & & \\
\cline { 2 - 3 } Subscales & $\begin{array}{c}\text { Low Score Group } \\
(n=628)\end{array}$ & $\begin{array}{c}\text { High Score Group } \\
(n=554)\end{array}$ & $t$ value & $p$ value \\
\hline Organizational Role Adaptability & $2.31 \pm 1.00$ & $2.87 \pm 1.04$ & -9.28 & 0.00 \\
Workload & $3.82 \pm 0.95$ & $4.30 \pm 0.78$ & -9.24 & 0.00 \\
Nurse-Patient Relationship & $3.17 \pm 0.91$ & $3.58 \pm 0.87$ & -8.01 & 0.00 \\
Income and Respect & $3.67 \pm 0.94$ & $4.07 \pm 0.82$ & -7.78 & 0.00 \\
Self-Efficacy & $2.01 \pm 0.77$ & $2.51 \pm 0.91$ & -10.18 & 0.00 \\
Responsibility & $3.49 \pm 0.80$ & $3.65 \pm 0.76$ & -3.48 & 0.00 \\
\hline
\end{tabular}

\section{General Discussion}

This study aimed to develop a new and practical scale to investigate the workplace stressor of Chinese nurses, which adapt the context of Chinese culture. The survey was performed on nurses from different administrative divisions in China, to explore the factor composition of nurse work stressors, and examine the reliability and validity of this scale. As a result, a scale (i.e. CNWSS-30), consisted of 6 factors (organizational role adaptability, workload, nurse-patient relationship, income and respect, self-efficacy, responsibility), was obtained.

Among these 6 factors, organizational role adaptability, workload, nurse-patient relationship and self-efficacy are highly similar with the results of previous researches (e.g. Gray-Toft \& Anderson, 1981; Wheeler \& Riding, 1994; Li \& Liu, 2000; Yu, 2007; Yuan et al., 2007; Wu, 1993). Relationships in nursing workplace, workload and self-ability were communal stressors existed, unaffected by region and time. Due to the particularity of nursing, these kinds of stressors possess cross cultural consistency. For instance, many studies suggested as the demand for high-quality health care is increasing, nurses should provide more, better, broader services for the patients. In addition, the growth of medical technology pushes new nurses to constantly learn unique skills (Yang, 2017). Furthermore, nurses face the interpersonal communication not only with the patients, but also with the colleagues, so the stress is anticipated.

Compared with other scales, CNWSS-30 firstly added 2 new factors (income and respect, responsibility). Salary satisfaction and the degree of social respect for nurses are always lower than doctors, and nurses have higher turnover intention (e.g. Pereira, Matos, \& Azevedo, 2014; Wang et al., 1999). In developing countries, nurses suffered from heavier workload, greater responsibility, lower salary and lower satisfaction than developed countries' nurses (e.g. Dong \& Qin, 2014; Yang, 2017). Thus, income and respect, responsibility, these kinds of stressors were more unique to the developing countries.

Work environment items are always associated with "bad environment", "insufficient equipment in the work" and so on. However, there were not work environment relevant items in CNWSS-30. This may due to the selected samples. 
All participants were from large 3a hospitals with better medical condition. In addition, with the social and economic development, there is a qualitative leap forward in hospital environment, and work environment may be no longer regard as a stressor for nursing workers.

In this study, all of the obtained parameters (e.g. internal consistency, test-retest reliability and the result of CFA) demand the requires of psychometrics. And the results of ATQ-30 demonstrated that the high score group had higher score at all six subscales of CNWSS than the low score group, which was consistent with previous studies (Lu, Siu, \& Cooper, 1997).

\section{Conclusion}

In present study, we developed and validated a 30 -item scale that measures nurse stressors in China. This scale is based on Chinese national conditions and culture context, and the results demonstrated that the CNWSS-30 has good reliability and validity. It can be used to measure the workplace stressor for Chinese nurse. In this study, all of the participants were from the large $3 \mathrm{~A}$ hospitals. In future study, we will select more other class hospitals and small-scale hospitals to test adaption of CNWSS-30.

\section{Conflicts of Interest}

The authors declare no conflicts of interest regarding the publication of this paper.

\section{References}

Applebaum, D., Fowler, S., Fiedler, N., \& Osinubi, O. Y. (2010). The Impact of Environmental Factors on Nursing Stress, Job Satisfaction, and Turnover Intention. Journal of Nursing Administration, 40, 323-328. https://doi.org/10.1097/NNA.0b013e3181e9393b

Arnetz, J. E., Sudan, S., Goetz, C., Count, S., \& Arnetz, B. (2019). Nurse Work Environment and Stress Biomarkers: Possible Implications for Patient Outcomes. Journal of Occupational and Environmental Medicine, 61, 676-681. https://doi.org/10.1097/JOM.0000000000001642

Cheng, Q. M., Liu, D. M., Wu, Y., \& Lu, J. J. (2015). Validation of Employee Assistance Program in Occupational Stress Management for Nurses. Journal of Nurses Training, 30, 688-690. (In Chinese)

Cimiotti, J. P., Aiken, L. H., Sloane, D. M., \& Wu, E. S. (2012). Nurse Staffing, Burnout, and Health Care-Associated Infection. American Journal of Infection Control, 40, 486-490. https://doi.org/10.1016/j.ajic.2012.02.029

Dong, Y. Y., \& Qin, Q. W. (2014). Scale of Medical Staff Resilience and Its Characteristics Research. Chongqing: Southwest University.

Gray-Toft, P., \& Anderson, J. G. (1981). Stress among Hospital Nursing Staff: Its Causes and Effects. Social Science \& Medicine. Part A: Medical Psychology \& Medical Sociology, 15, 639-647. https://doi.org/10.1016/0271-7123(81)90087-0

Hollon, S. D. \& Kendall, P. C. (1980). Cognitive Self-Statements in Depression: Development of an Automatic Thoughts Questionnaire. Cognitive Therapy and Research, 4, 383-395. https://doi.org/10.1007/BF01178214 
Khamisa, N., Peltzer, K., \& Oldenburg, B. (2013). Burnout in Relation to Specific Contributing Factors and Health Outcomes among Nurses: A Systematic Review. International Journal of Environmental Research and Public Health, 10, 2214-2240. https://doi.org/10.3390/ijerph10062214

Lee, J. S., \& Akhtar, S. (2011). Effects of the Workplace Social Context and Job Content on Nurse Burnout. Human Resource Management, 50, 227-245. https://doi.org/10.1002/hrm.20421

Li, X. M., \& Liu, Y. J. (2000). Job Stressors and Burnout among Staff Nurses. Chinese Journal of Nursing, 2, 4-8.

Lu, L., Siu, O. L., \& Cooper, C. L. (1997). Occupational Stress in Clinical Nurses. Counselling Psychology Quarterly, 10, 39-50. https://doi.org/10.1080/09515079708251410

Mcvicar, A. (2003). Workplace Stress in Nursing: A Literature Review. Journal of Advanced Nursing, 44, 633-642. https://doi.org/10.1046/j.0309-2402.2003.02853.x

National Institute for Occupational Safety and Health (1999). What Is Job Stress. https://www.cdc.gov/niosh/docs/99-101/

National Institute for Occupational Safety and Health (2008). Exposure to Stress: Occupational Hazards in Hospitals. http://www.cdc.gov/niosh/docs/2008-136/

Pereira, I., Matos, A., \& Azevedo, A. (2014). Portuguese Version of The Automatic Thoughts Questionnaire-Revised: Study of Its Psychometric Properties and Relationship with Depressive Symptomatology in Adolescents. Psicologia Saúde \& Doença, 15, 37-47.

Sun, F., \& Wen, Z. X. (2013). Surveys of Current Situation of Mental Health and Sources of Stress of Nurse Working in Department of Oncology. Journal of Qingdao University Medical Sciences, 49, 59-61+64.

Tong, H. J. (2002). Surveying and Expecting: Three Majar Test Technologies in Psychology. Journal of Nanjing Normal University (Social Science Edition), 3, 81-88.

Wang, X. D., Wang, X. L., \& Ma, H. (1999). Handbook of Mental Health Scale for the Updated Version. Chinese Mental Health Journal, 12, 215-217.

Wang, Y., \& Meng, X. Z. (2007). Primary Modification of Chinese Nurse Job Stressors Questionnaire. Chinese Journal of Clinical Psychology, 2, 129-131.

Wheeler, H., \& Riding, R. (1994). Occupational Stress in General Nurses and Midwives. British Journal of Nursing, 3, 527-534. https://doi.org/10.12968/bjon.1994.3.10.527

Wu, I. C. (1993). Designing Scale for Frequency and Perception of Job Stress of Clinical Nurses. Nursing Research (Taiwan), 2, 113-126.

Yang, P. (2017). Development of a Career Stress Scale for Hospital Nurses: Implications for Workplace Counseling. Journal of Employment Counseling, 54, 156-167. https://doi.org/10.1002/joec.12064

$\mathrm{Yu}, \mathrm{H}$. (2007). Analysis on Reliability and Validity of Chinese Nurses Stressor scale. Chinese Nursing Research, 23, 2090-2093.

Yuan, Y., Yu, H., \& Xu, X. F. (2007). Reliability and Validity of the Chinese Nursing Stressor Scale. Chinese Journal of Nursing Education, 5, 195-198. 